Gynäkologe 2020 $53: 784-788$

https://doi.org/10.1007/s00129-020-04685-1

Online publiziert: 2. Oktober 2020

(c) Der/die Autor(en) 2020

\section{Redaktion}

R. Felberbaum, Kempten

R. Kreienberg, Mainz

B. Ramsauer, Berlin

Johannes Brandner' ${ }^{1}$ Martin Wald ${ }^{1}$ Lorenz Auer-Hackenberg ${ }^{1}$ Judith HuberKatamay $^{2} \cdot$ Claudius Fazelnia $^{2} \cdot$ Monika Edelbauer-Wechselberger $^{3}$

' Universitätsklinik für Kinder- und Jugendheilkunde der PMU, Division für Neonatologie, Uniklinikum Salzburg, Salzburg, Österreich

${ }^{2}$ Universitätsklinik für Gynäkologie und Geburtshilfe der PMU, Uniklinikum Salzburg, Salzburg, Österreich

${ }^{3}$ Universitätsklinik für Kinder- und Jugendheilkunde der PMU, Uniklinikum Salzburg, Salzburg, Österreich

\title{
Normale Lungenentwicklung bei Einlingsgravidität mit bilateraler Nierenagenesie - ein Paradoxon?
}

füllung werden besprochen. Es wird auch auf die Möglichkeit einer medizinisch indizierten Abruptio oder einer postnatalen Comfort-care-Versorgung des Kindes hingewiesen. Die Eltern entscheiden sich gegen einen Abbruch, aber auch gegen weitere Diagnostik. Routinekontrollen werden vereinbart. Mit 31/32 SSW besteht plötzlich ein FW-Depot, bis zur 34. SSW fast eine normale FW-Menge. Infolge der sonographischen Befundänderung und einer fetalen MRT (s. unten) wird gemeinsam mit den Eltern ein aktives Management beschlossen, Neonatologie und Kinderchirurgie werden mit einbezogen.

\section{Pränataldiagnostik}

\section{Sonographien}

\section{$19+3$ SSW \\ (• Abb. 1)}

Verdacht auf Nierenagenesie links und multizystische Niere rechts - Differenzialdiagnose (DD) beidseitige Nierenagenesie. Harnblase nicht gefüllt, Nierenarterien nicht darstellbar, schweres Oligohydramnion, hypertrophe Kardiomyopathie. Soweit beurteilbar, keine weiteren fetalen Auffälligkeiten.

\section{$30+6 u .32+2 S S W$ \\ (•Abb. 2)}

Unauffällige Dopplersonographie, etwas FW darstellbar. Magen und Harnblase nicht darstellbar. Nebennieren darstellbar, dopplersonographisch

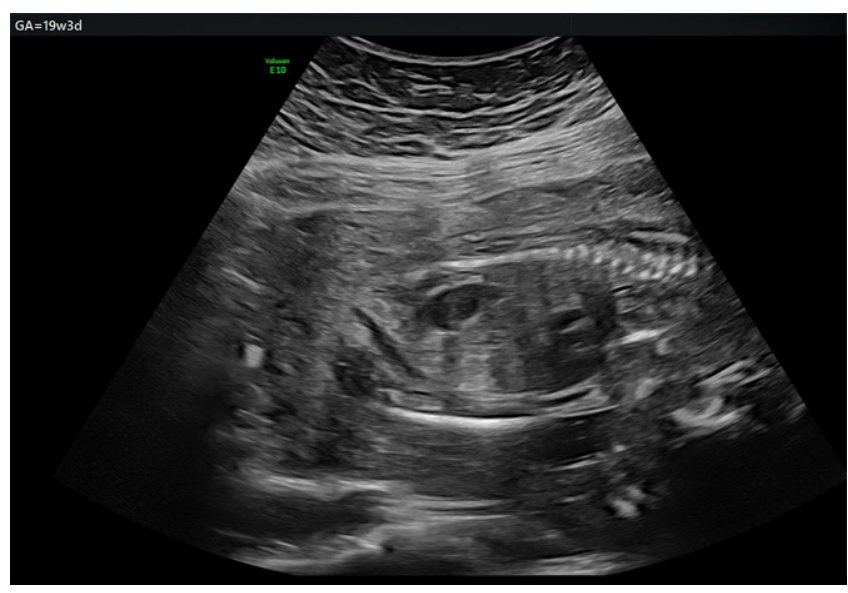

Abb. $1 \Delta$ Anhydramnion $19+3$ SSW

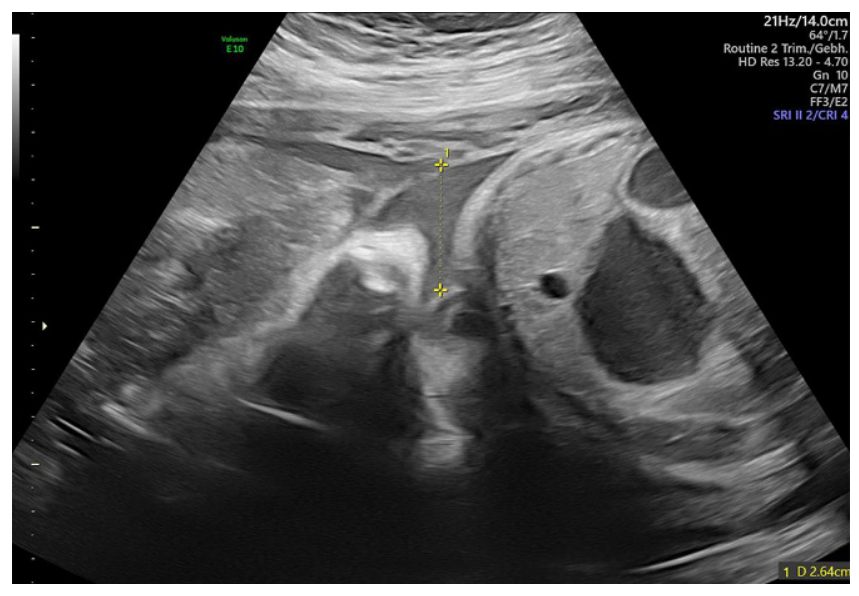

Abb. $2 \Delta 30+6$ SSW (Schwangerschaftswochen) FW(Fruchtwasser)-Depot 


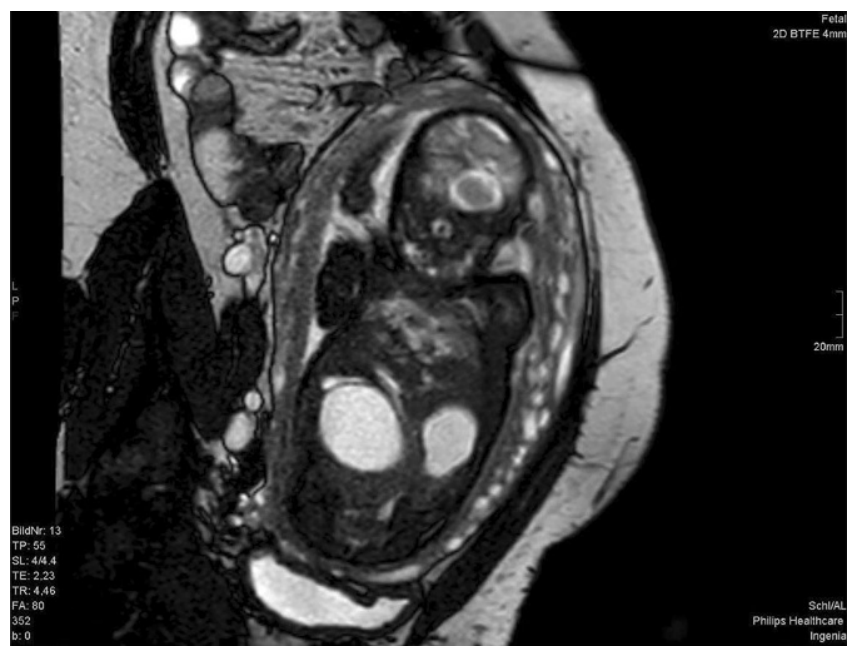

Abb. $3 \triangle$ MRT (Magnetresonanztomographie) $32+3$ SSW (Schwangerschaftswochen)

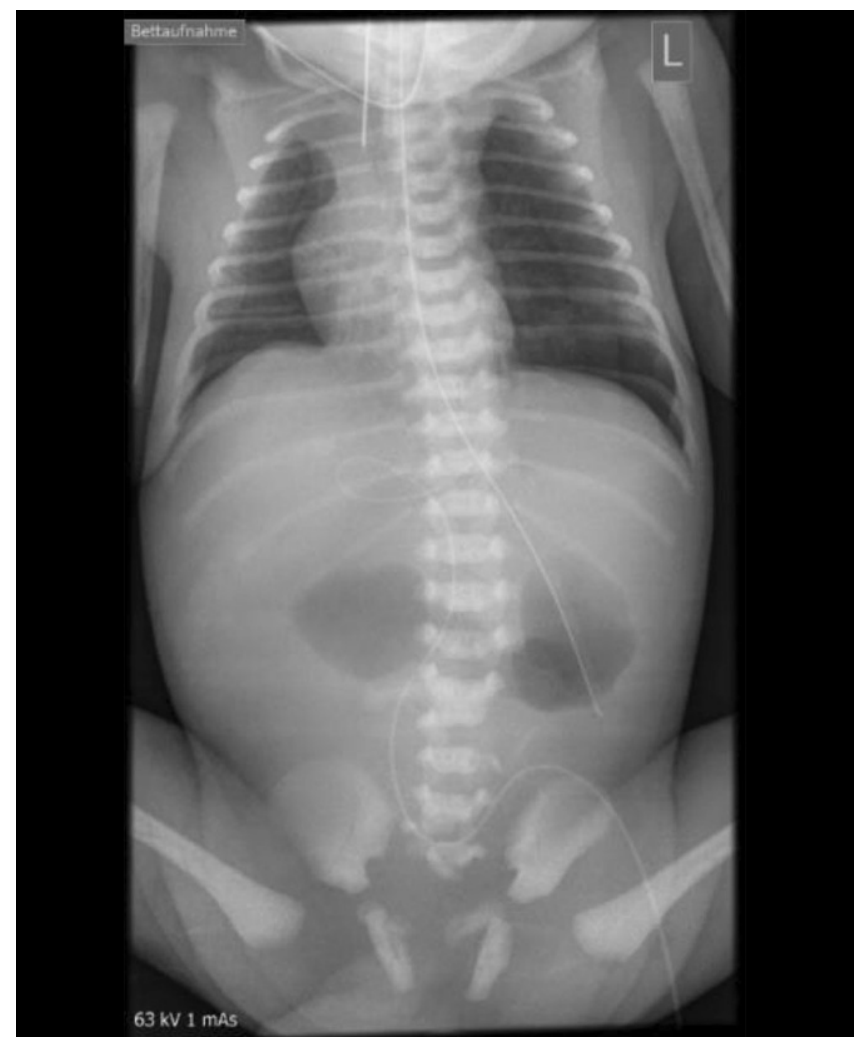

Durchblutung in Richtung beider Nierenbecken darstellbar. Im Bereich beider Nierenbecken große flüssigkeitsgefüllte Hohlräume DD Hydronephrose, DD Megaurether, kaudal eventuell kommunizierend. IUGR („intrauterine growth restriction“). Verdacht auf Fehlbildung im Urogenitaltrakt. Geschlechtschromosomen XY.

\section{$34+2$ SSW}

Erneut 2 große zystische Strukturen mit überwiegendem leerem, teils flottierendem echoreichen (Zelldetritus?) Inhalt. Kaudale Kommunikation beider Strukturen eindeutig darstellbar. FW im unteren Normalbereich. Magen und Harnblase sonographisch nicht darstellbar.

\section{Fetale MRT $32+3$ SSW}

\section{(• Abb. 3)}

Im Mittel-/Oberbauch zeigen sich beidseits mit mutmaßlicher ventralseitiger paramedianer Kommunikation 2 zystoide Strukturen mit $42 \times 27$ bzw. $30 \times 25 \mathrm{~mm}$. Kraniodorsal angrenzend angedeutetes bandförmiges Parenchym mit ebenfalls Kommunikation über die Mittellinie. Insgesamt besteht der Verdacht auf eine Hufeisenniere mit gemeinsamen, kommunizierenden Nierenbecken und mutmaßlich hoher ureteraler Obstruktion. Eine umschriebene Struktur mit Flüssigkeitsmarkierung median im kleinen Becken mit $8 \times 4 \mathrm{~mm}$ wird als entleerte Harnblase gedeutet. Keine

Abb. $4 \Delta$ Röntgenaufnahme des Abdomens am 1. Lebenstag (LT)

eindeutige flüssigkeitsmarkierte Magenblase, wahrscheinlich kompressionsbedingt. Oligohydramnion.

Lunge beidseits angelegt. Soweit technisch möglich wird das Lungenvolumen in mehreren Ebenen gemessen mit Werten zw. $13 \mathrm{ml}$ (transversale Schichtführung) und $22 \mathrm{ml}$ (koronar).

\section{Procedere}

Eine Entbindung mit $37+0$ SSW wird geplant, bei vorzeitigem Blasensprung und pathologischem CTG (Kardiotokogramm) muss aber bereits mit $34+4$ SSW eine Sectio caesarea durchgeführt werden. Das Kind zeigt eine gute postnatale Adaptation (APGAR 6/8/8/8; Geburtsgewicht Perzentile 3, Länge und Kopfumfang Perzentile 10, NS-Astrup unauffällig). Die respiratorische Stabilisierung über die folgenden Tage ist schwieriger. Nach $2 \times$ intratrachealer Surfactantgabe, Anwendung von iNO (inhalativem Stickstoffmonoxid), Sildenafil, Katecholaminen in niedriger Dosierung sowie Entlastung eines einseitigen Pneumothorax zeigt das Kind aber eine suffiziente Spontanatmung bei nur geringer Unterstützung mittels Nasenbrille bei Raumluft. Außer einer Analatresie finden sich in der klinischen Untersuchung keine Hinweise für Dysmorphien. Bei zu der u.a. postnatalen Diagnostik passenden persistierenden Anurie wird im Alter von 3 Tagen eine Peritonealdialyse etabliert [2]. Aufgrund des pränatal wie postnatal insgesamt unklaren Verlaufes wird ein MRT geplant, sobald es der Allgemeinzustand des Kindes erlaubt.

\section{Röntgenaufnahme am \\ 1. Lebenstag}

\section{(• Abb. 4)}

Bild wie bei „double bubble“ mit luftmarkiertem Magenfundus und Bulbus duodeni rechts paravertebral. Sonst keineluftmarkierten intestinalen Strukturen abgrenzbar. Die Brustwirbelkörper imponieren regulär. Ausgehend von 12 Rippenpaaren Bild wie bei Blockwirbelbildung von L4/L5. Soweit beurteilbar auch die sakralen Wirbelkörper atypisch mit 
nur 3 ossifiziert abgrenzbaren, irregulär begrenzten Wirbelkörpern.
Sonographie Abdomen am

\section{Lebenstag}

Kein Nachweis von orthotopen Nierengewebe oder einer Hufeisenniere, Nierenlogen beidseits darmschlingengefüllt. Kein Hinweis auf dilatierte Nierenbe- ckenkelchsysteme - Bild wie bei Nierenagenesie beidseits. Eine zur kontrahierten, entleerten Harnblase passende Struktur im kleinen Becken. Minimal Aszites. Bei Vorliegen einer Analatresie multiple mekoniummarkierte Darmstrukturen im kleinen Becken.

Wie lautet Ihre Diagnose?

\section{》) Diagnose: Bilaterale \\ Nierenagenesie mit normaler Lungenentwicklung durch ausreichende FW-Menge bei zusätzlicher Duodenalatresie}

\section{MRT Schädel, Abdomen, Becken am 10. Lebenstag}

\section{(• Abb. 5)}

Schädel der SSW entsprechend unauffällig. Nierenagenesie beidseits. Analatresie. Hinweise für eine Duodenalatresie und Malrotation des Gastrointestinaltraktes. Zeichen einer weiteren Dünndarmstenose im Unterbauch/ Mittelbauch median und rechts. Kaudales Regressionssyndrom mit partieller Sakralagenesie und lumbaler Wirbelkörperfehlbildung. Abnormer Befund der Aorta abdominalis und der arteriellen Beckenstrombahn.

\section{Interpretation der Bildgebung im prä-/postnatalen Verlauf (. Abb. 3 und 4)}

Die initiale Verdachtsdiagnose der bilateralen Nierenagenesie kann klinisch und im MRT eindeutig bestätigt werden. Das Korrelat für das „double bubble sign“ im Röntgen am ersten Lebenstag (LT) stellt sicherlich der MRT-Befund der Duodenalatresie dar. Die als „Hufeisenniere mit hoher ureteraler Obstruktion" interpretierten zystoiden Strukturen in der fetalen MRT entsprechen mit großer Wahrscheinlichkeit dem „double bubble sign“ im postnatalen Röntgen mit dann (partieller) Luftfüllung der dilatierten Darmabschnitte.

\section{Postnataler Verlauf}

Die Befunde des Kindes und das weitere Procedere werden wiederholt interdisziplinär (Geburtshilfe, Neonatologie, Kindernephrologie, Kinderchirurgie) und mit den Eltern besprochen. Eine chirurgische Sanierung der multiplen

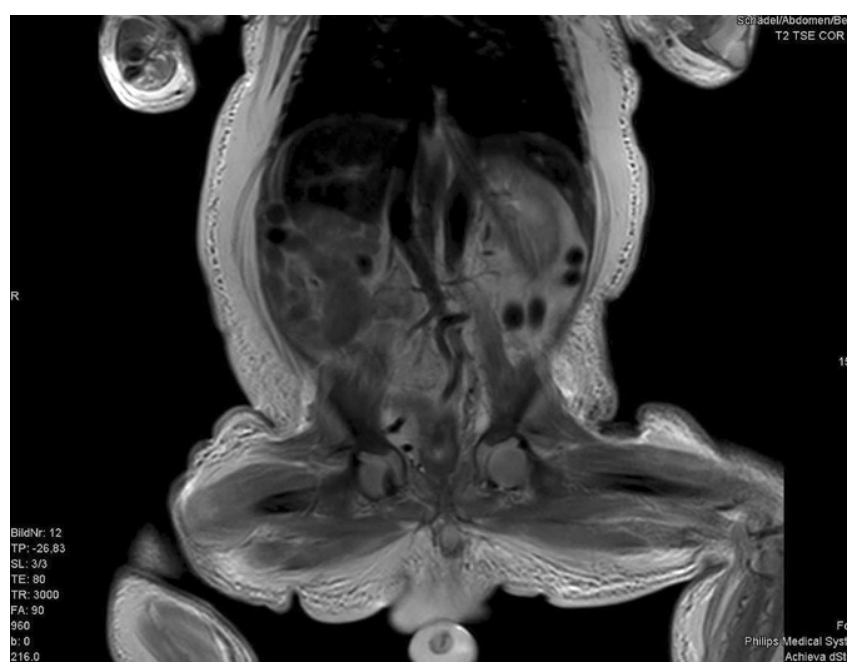

Abb. $5<$ Magnetresonanztomographie am 10. Lebenstag intestinalen und der beim Befund eines kaudalen Regressionssyndroms vermuteten urogenitalen Malformationen hätte eine deutlich eingeschränkte Prognose speziell unter laufender Peritonealdialyse. Die Möglichkeit einer Hämodialyse wird diskutiert. Letztendlich wird aber, auch nach dem dezidierten Wunsch der Eltern, eine Palliativbetreuung mit Beendigung der Nierenersatztherapie beschlossen. Am 14. LT, am Tag nach Beendigung der Peritonealdialyse und der anhaltend nur gering notwendigen Atemunterstützung, verstirbt das Kind friedlich im Beisein seiner Eltern. Eine psychologische Betreuung und Begleitung der Eltern erfolgt kontinuierlich von der Phase der pränatalen Diagnostik bis nach Ableben des kleinen Patienten.

\section{Diskussion}

Eine beidseitige Nierenagenesie führt üblicherweise zu Oligo-/Anhydramnie mit Folgen wie Lungenhypoplasie oder Potter-Sequenz [1, 3]. Wenige Fallberichte von bilateraler Nierenagenesie mit normaler Lungenfunktion bei einem von monoamnioten Zwillingen [3, 4] vermuten, dass die FW-Menge in der gemeinsamen Fruchtblase vom gesunden Zwilling aufrechterhalten wird. Der bisher einzige publizierte Fall einer Einlingsgravidität betrifft ebenfalls ein Kind mit Duodenalatresie und urorektaler Fehlbildung [1].

In der auch von uns primär vertretenen Theorie wird angenommen, dass die FW-Reduktion durch fetales Verschlucken aufgrund der Duodenalatresie ineffektiv war und damit ein ausreichendes FW-Volumen aufrechterhalten wurde [1]. In diese Überlegungen miteinzubeziehen ist allerdings auch eine Beteiligung von intramembranösen "pathways“ 
der Amnionmembranen an der Regulation der FW-Menge $[1,5]$ - insbesondere vor dem bekannten Hintergrund, dass nur ein Drittel der Feten mit Ösophagusatresie und zwei Drittel der Feten mit Duodenal- oder proximaler Jejunalatresie ein Polyhydramnion entwickeln [1].

Eine andere Theorie vermutet eine Störung der Gefäßversorgung im Becken später in der Schwangerschaft, die zur „Nierenagenesie“ und Atrophie von Beckenorganen [1] führt - eine Überlegung, welche die Pathophysiologie unseres $\mathrm{Pa}$ tienten vor dem Hintergrund der abnormen MRT-Befunde von Aorta abdominalis und Beckenarterien zumindest zum Teil erklären würde. Pränatal noch erkennbare renale Strukturen wären dann zum Bild einer postnatalen Nierenagenesie degeneriert. Die Anhydramnie bis zum zweiten Trimenon wäre eventuell durch eine gestörte Diffusion zwischen Fetus und FW erklärbar $[1,5]$.

\section{Fazit für die Praxis}

- Die aufgrund der Lungenhypoplasie bisher als infaust eingestufte Prognose bei bilateraler Nierenagenesie ist zu hinterfragen

- Abhängig von Komorbiditäten ist inzwischen auch eine schon in der Neonatalperiode begonnene Nierenersatztherapie mit dann frühzeitiger Nierentransplantation mit einem vielversprechenden Outcome verbunden

- Jedenfalls ist eine individuelle Diskussion des prä- und postnatalen Managements im multidisziplinären Setting zur fundierten Beratung der Eltern notwendig

\section{Korrespondenzadresse}

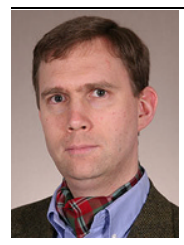

Dr. med.

Johannes Brandner

Universitätsklinik für Kinderund Jugendheilkunde der PMU, Division für Neonatologie, Uniklinikum Salzburg Müllner Hauptstr. 42, 5020 Salzburg, Österreich j.brandner@salk.at
Funding. Open access funding provided by Paracelsus Medical University.

\section{Einhaltung ethischer Richtlinien}

Interessenkonflikt. J. Brandner, M. Wald, L. Auer-Hackenberg, J. Huber-Katamay, C. Fazelnia und M. Edelbauer-Wechselberger geben an, dass kein Interessenkonflikt besteht.

Für diesen Beitrag wurden von den Autoren keine Studien an Menschen oder Tieren durchgeführt. Für die aufgeführten Studien gelten die jeweils dort angegebenen ethischen Richtlinien. Für Bildmaterial oder anderweitige Angaben innerhalb des Manuskripts, über die Patienten zu identifizieren sind, liegt von innen und/oder ihren gesetzlichen Vertretern eine schriftliche Einwilligung vor.

Open Access. Dieser Artikel wird unter der Creative Commons Namensnennung 4.0 International Lizenz veröffentlicht, welche die Nutzung, Vervielfältigung, Bearbeitung, Verbreitung und Wiedergabe in jeglichem Medium und Format erlaubt, sofern Sie den/die ursprünglichen Autor(en) und die Quelle ordnungsgemäß nennen, einen Link zur Creative Commons Lizenz beifügen und angeben, ob Änderungen vorgenommen wurden.

Die in diesem Artikel enthaltenen Bilder und sonstiges Drittmaterial unterliegen ebenfalls der genannten Creative Commons Lizenz, sofern sich aus der Abbildungslegende nichts anderes ergibt. Sofern das betreffende Material nicht unter der genannten Creative Commons Lizenz steht und die betreffende Handlung nicht nach gesetzlichen Vorschriften erlaubt ist, ist für die oben aufgeführten Weiterverwendungen des Materials die Einwilligung des jeweiligen Rechteinhabers einzuholen.

Weitere Details zur Lizenz entnehmen Sie bitte der Lizenzinformation auf http://creativecommons.org/ licenses/by/4.0/deed.de.

\section{Literatur}

1. George L, Manitim W, Sharma J (2017) A singleton infant with bilateral renal agenesis and normal pulmonary function. Hindawi Case Rep Pediatr 2017:1710371

2. van Stralen KJ et al (2014) Survival and clinical outcomes of children starting renal replacement therapy in the neonatal period. Kidney Int 86:168-174

3. Klingler G, Merlo P, Aloni D, Maayan A, Sirota L (2007) Normal pulmonary function in a monoamniotic twin discordant for bilateral renal agenesis: report and review. Am J Med Genet 73(1):76-79

4. Perz-Brayfield MR, Kirsch AJ, Smith EA (2004) Monoamniotic twin discordant for bilateral renal agenesis with normal pulmonary function. Urology 64(3):589

5. Underwood MA, Gilbert WM, Sherman MP (2005) State of the art-amniotic fluid: not just fetal urine anymore. J Perinatol 25:341-348 
Hier steht eine Anzeige.

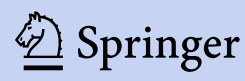

